tissue disease (MCTD), undifferentiated connective tissue disease (UCTD) and healthy controls $(\mathrm{HC})$ will be analysed to identify clinically relevant clusters.

Methods: A European multi centre, non-randomised, cross-sectional clinical study was conducted in 18 sites and 9 countries. Collection of OMIC data including genetic, epigenomic, transcriptomic (from peripheral blood and from isolated cells), flow cytometry, metabolomics and proteomic in plasma and urine, exosome analysis and classical serology (antibodies and autoantibodies) was organised. Novel and innovative methodologies including fine flow cytometry were conducted. Quality procedures were established to ensure standardisation of samples collection, processing, transportation and storage. Techniques were validated to ensure reproducibility of analyses. Unsupervised bioinformatics and biostatistics approaches will be applied.

Results: Recruitment started in December 2014 and ended in October 2017. A total of 2656 participants were recruited: 377 RA, 470 SLE, 402 SSc, 385 SjS, 99 MTCD, 106 PAPs, 166 UCTD patients and $651 \mathrm{HCs}$. Median age was between 46 and 59 years and was consistent with each disease onset peak. $97 \%$ of the population was Caucasian. Most of the patients were treated with standard of care therapies and less than $10 \%$ were on biologics. OMICs and bioinformatics analyses are on-going.

Conclusions: We have established one of the largest collaborative multi-OMICs studies from patients with SADs. The most important challenge is now the integration of all these novel data to support hypothesis-free, machine learning-led analytical protocols. It is expected that the integration of data from affected patients, in comparison with well-matched controls, will provide new biomarker-led descriptions of clusters of potentially etiologically distinct disease entities.

Acknowledgements: This work has received support from the EU/EFPIA/Innovative Medicines Initiative Joint Undertaking PRECISESADS grant n 115565 Disclosure of Interest: None declared

DOI: 10.1136/annrheumdis-2018-eular.4625

\section{AB1373 URINARY PROTEIN PROFILE COMPARISON BETWEEN SLE PATIENTS WITH AND WITHOUT RENAL INVOLVEMENT}

N. Rivera ${ }^{1}$, E. Ruiz ${ }^{1}$, E. Úcar ${ }^{1}$, M.L. García Vivar ${ }^{1}$, F. Elortza ${ }^{2}$, M. Azkargorta ${ }^{2}$, O. F. Berrizbeitia ${ }^{1}$, E. Galindez ${ }^{1}$, I. Torre ${ }^{1}$, A. Intxaurbe ${ }^{1}$, J. Blanco ${ }^{1}$, C. Pérez ${ }^{1}$, M. J. Allande ${ }^{1}$, O. Ibarguengoitia ${ }^{1}$, I. Calvo ${ }^{1}$, E. Guerrero ${ }^{1} .{ }^{1}$ Rheumatology, Basurto University Hospital; ${ }^{2}$ Proteomic, CIC bioGUNE, Bilbao, Spain

Background: Lupus nephropathy (NL) is an important cause of morbidity and mortality in patients with Systemic Lupus Erythematosus (SLE). The objective of the renal biopsy is to determine the type of glomerulonephritis that the patient presents to direct treatment. Considering that it is a specialised technique and not risk free, a proteomics study is proposed to determine biomarkers that help us to differentiate patients diagnosed with SLE with and without renal involvement.

Objectives: To determine if there is a different pattern of proteins between patients diagnosed with SLE with and without renal involvement.

Methods: We selected 12 patients diagnosed with SLE with renal involvement and 14 patients diagnosed with SLE without renal involvement. There were no differences between groups according to race, gener and age. The patients were classified as high, low or negative level of proteinuria in the urine. A 24 hour urine sample was obtained for analysis.

Results: We have done a Principal Component Analysis (PCA) where we can see differences between samples from patients who have high level of proteinuria in 24 hours and patients who have not renal involvement. Patients with positive proteinuria but not high level are a little confuse figure 1 .

A total of 292 proteins (identified with at least two peptides with a FDR $<1 \%$ ) were quantified and further considered in the analysis. The Student's T-test analysis reflected the differential presence of 147 proteins $(p<0.01)$. Of these, 130 were less abundant in the urine of the patients with renal damage, whereas 17 showed the opposite pattern, being more abundant in the patients with affected renal function.

Consistent with the nature of the sample, the Gene Ontology (GO analysis) of the whole list of identified proteins revealed the presence of extracellular (277 proteins, $p=2.25 \mathrm{E}-171)$ and secretion-related proteins (49 proteins, $\mathrm{p}=1.1 \mathrm{E}-09$ ), among others. Proteins related to defensive processes were prominent among them. Interestingly, the subset of proteins whose abundance increases upon renal damage is comprised of typical highly-abundant serum proteins. These proteins render a large number of peptides, suggesting they are very abundant. This protein pattern may reflect the higher albuminuria characteristic of patients with affected renal function. On the other hand, a number of proteins became significantly less abundant upon renal damage. The presence of highly abundant serum proteins in the urine of patients with compromised renal function may explain this phenomenon, since this will provoke a dramatic reduction in the relative abundance of the proteins already present in their urine.

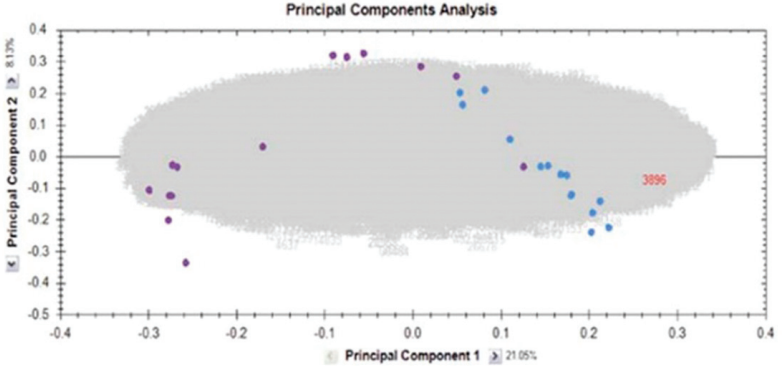

Abstract AB1373 - Figure 1

Conclusions: A different protein pattern is observed between the two groups of patients, so in a more detailed study we can indicate if some of these can serve as prognostic markers for this type of patients.

Disclosure of Interest: None declared

DOI: 10.1136/annrheumdis-2018-eular.3720

\section{AB1374 1 PERFORMANCE OF EQ-5D, RAPID-3 AND HADS SCALES IN THE ASSESSMENT OF QUALITY OF LIFE AND FUNCTIONAL STATUS IN PATIENTS WITH ERYTHEMA NODOSUM}

N. Savushkina, V. Amirdjanova, O. Egorova, B. Belov. V. A. Nasonova Research Institute of Rheumatology, Moscow, Russian Federation

Background: Erythema nodosum (EN) is the typical example of a mostly septal panniculitis with no vasculitis. Sarcoidosis and streptococcal infection are consid ered to be the most common etiological factors. There are no special scales available for practitioners to assess the efficacy of EN treatment in spite of high disease prevalence in general population. Sensitivity, specificity and validity of EQ-5D, RAPID-3 and HADS questionnaires have been demonstrated in other rheumatic diseases.

Objectives: The aim of this study is to evaluate the psychometric properties of EQ-5D, RAPID-3 and HADS scales in EN patients.

Methods: The study included 47 patients (45 females, 2 males) aged 37,6 $\pm 11,2$ y with mean EN duration $2,5^{1 ; 6}$ months who were on the record at $\mathrm{V}$. A Nasonova Research Institute of Rheumatology during 2013-2017 yy. All patients filled in EQ-5D, RAPID-3 and HADS questionnaires at baseline (first visit) and 12 months later. The sensitivity of the questionnaires was assessed by comparing obtained scores with patient's response to therapy until achievement of nodular regression at the time of dynamic examination. The construct validity was determined using a correlation analysis with "external criteria", such as presence of artralgia/arthritis, palpatory tenderness of nodules assessed with VAS scale, ESR and CRP levels.

Results: Complete nodular regression was achieved in 39 patients. Positive post-treatment changes (nodular regression) correlated with improved EQ-5D (EQ-5D-index $-p=0,005$, EQ-5D-VAS $-p=0,009$ ) and HADS-anxiety subscale $p=0,02$. No significant association was found for other questionnaires with $p$ value $=0,11$ for RAPID-3 and $p=0,69$ for HADS-depression scale. Moderate correlation was established at control visit after 12 months (Mo12) between EQ-5D index and nodular tenderness to palpation ( $r=-0,49, p=0,0001)$, between $E Q-5 D$ index and ESR value $(r=-0,55, p=0,0016)$, as well as between general health status assessed by VAS and nodular tenderness to palpation at baseline visit $(r=-$ $0,56, p=0,0001$ ), indicating close association of data obtained by these assess ment tools with objective physical and laboratory findings. There was also moder ate correlation between functional RAPID-3 scores and nodular tenderness to palpation at Mo12 $(r=0,37, p=0,0172)$ and ESR level $(r=0,52, p=0,0002)$. These data demonstrate close association of assessment scales performance with objective health status. Moderate correlation was established for HADS-depression subscale scores with nodular tenderness to palpation in 12 moth after initiation of treatment $(r=0,41, p=0,0077)$ and CRP value $(r=0,34, p=0,025)$. There were no additional statistically significant correlations for RAPID-3 and HADS subscales with clinical and laboratory findings.

Conclusions: EQ-5D questionnaire is a valid and sensitive tool for assessment of quality of life in EN patients.

Acknowledgements: The study had no sponsorship.

Disclosure of Interest: None declared

DOI: 10.1136/annrheumdis-2018-eular.2270 


$\mathrm{AB} 1375$

RELIABILITY AND VALIDITY OF TURKISH VERSION OF SHORT FORM OF THE SOCIAL ROLE PARTICIPATION QUESTIONNAIRE (S-SRPQ) IN PATIENTS WITH ANKYLOSING SPONDYLITIS

Y. Akyol ${ }^{1}$, Y. Ulus ${ }^{1}$, Y. Terzi ${ }^{2}$, A. Bilgici ${ }^{1}$, O. Kuru ${ }^{1} .{ }^{1}$ Physical Therapy and Rehabilitation; ${ }^{2}$ Statistics, Ondokuzmayıs university, Samsun, Turkey

Background: Ankylosing spondylitis (AS) is a chronic inflammatory rheumatic disease with a usual onset in the third decade of life, when persons are committed to various social roles that adults fulfil. ${ }^{1}$ When evaluating the outcomes of clinical care, social role participation is an increasingly important outcome, especially when considering diseases with substantial limitations in physical functioning such as inflammatory rheumatic diseases. ${ }^{2}$ The Short Form of the Social Role Participation Questionnaire (s-SRPQ) is a questionnaire which was developed to assess effect of ankylosing spondylitis (AS) on participation. ${ }^{2}$

Objectives: This study aims to evaluate the reliability and validity of the Turkish version of the s-SRPQ in Turkish patients with AS.

Methods: The Turkish version of S-SRPQ questionnaire was obtained after a translation and back translation process. The study sample included $100 \mathrm{AS}$ patients (59 males, 41 females; mean age $42.00 \pm 11.00$ years; range 19 to 69 years). To assess the test-retest reliability of the Turkish s-SRPQ, the questionnaire was reapplied 15 days after the first interview (interclass correlation coefficient, ICC). Cronbach's alpha (a) was used to evaluate the internal consistency. The s-SRPQ was compared with Short Form-36 survey (SF-36), Ankylosing Spondylitis Quality of Life questionnaire (ASQoL), Bath Ankylosing Spondylitis Disease Activity Index (BASDAI), Bath Ankylosing Spondylitis Functional Index (BASFI), and Satisfaction With Life Scale (SWLS) for convergent validity. The internal structure of s-SRPQ was examined by factor analysis.

Results: For s-SRPQ/experienced physical difficulties; the individual item ICC ranged from 0.78 to 1.00 and Cronbach's alpha value ranged from 0.88 to 1.00 . For S-SRPQ/satisfaction with role performance; the individual item ICC ranged from 0.93 to 0.98 and Cronbach's alpha value ranged from 0.96 to 0.99 . KMO values was determined as 0.90 and 0.89 in the s-SRPQ/experienced physical difficulties and s-SRPQ/satisfaction with role performance, respectively. Bartlett's test of sphericity had a $p<0.001$. The Turkish version of $s-S R P Q / e x p e r i e n c e d$ physical difficulties scores negatively correlated with the SWLS and SF-36 sub-parameters scores $(p<0.01)$. There were also strong positive correlations between $s$-SRPQ/ experienced physical difficulties scores and BASDAI, BASFI, and ASQoL scores $(p<0.01)$. The $S R P Q /$ satisfaction with role performance scores positively correlated with the SWLS and SF-36 sub-parameters scores $(p<0.01)$. There were also strong negative correlations between s-SRPQ/experienced physical difficulties scores and BASDAI, BASFI, and ASQoL scores $(p<0.01)$.

Conclusions: Turkish version of s-SRPQ has good comprehensibility, internal consistency, and validity and is an adequate and useful instrument for the assessment of participation in Turkish patients with AS.

\section{REFERENCES:}

[1] Sieper J, Braun J, Rudwaleit M, Boonen A, Zink A (2002) Ankylosing spondylitis: an overview. Ann Rheum Dis 61(Suppl 3):8-18

[2] Oude Voshaar M, van Onna M, van Genderen S, van de Laar M, van der Heijde D, Heuft L, et al. (2016) Development and Validation of a Short Form of the Social Role Participation Questionnaire in Patients with Ankylosing Spondylitis. J Rheumatol 43(7):1386-92

Disclosure of Interest: None declared

DOI: 10.1136/annrheumdis-2018-eular.1198

\section{$\mathrm{AB} 1376$ \\ URINARY MONOCYTE CHEMOATTRACTANT PROTEIN 1 CANNOT DIFFERENTIATE BETWEEN HISTOLOGICAL CLASSES OF LUPUS NEPHRITIS}

R.K. Gone ${ }^{1}$, V. Dhir ${ }^{2}$, A. Sandhu ${ }^{2}$, A. Sharma ${ }^{2}$, M. Rathi ${ }^{3}$, R. Nada ${ }^{4}$, S. Sharma ${ }^{5}$ S.K. Sharma ${ }^{6}{ }^{1}$ Internal medicine, Post graduate institutite of medical education and research, Chandigarh; ${ }^{2}$ Internal medicine; ${ }^{3}$ Department of Nephrology; ${ }^{4}$ Department of Histopathology; ${ }^{5}$ Department of Biochemistry, Post Graduate Institute of Medical Education and Research; ${ }^{6}$ Internal medicine, Post Graduate Medical Education Education and Research, Chandigarh, India

Background: Lupus Nephritis, one of the commonest manifestation of SLE, seen in $60 \%$ of adult SLE patients at some point of time, carries poor prognosis when compared to those with no renal involvement. The natural course of the LN has recurrent flares, necessitating the need for early detection and treatment. Despite being the gold standard in diagnosing Lupus nephritis and its severity, renal biopsy is an invasive procedure with potential complications, and difficult to repeat. Hence, a novel biomarker, reflecting the disease activity and severity is needed to predict flare. Monocyte chemoattractant Protein 1, a chemokine produced locally during Lupus Nephritis flare, was shown in many previous studies as a promising biomarker

Objectives: We studied the role of Urinary MCP-1 as a biomarker of disease activity in LN and compare its value among different histological classes of Lupus nephritis

Methods: This is a case-control study conducted at a tertiary care centre in North India from July 2016 to December 2017. Cases were those patients undergoing renal biopsy satisfying the inclusion criteria set for SLE with $\operatorname{LN}(n=36)$. Controls were patients of SLE without active $L N$ [Control I-prior $L N(n=11)$ and Control IInever had LN $(n=15)]$. Urinary MCP-1 measurement was done using Sandwich ELISA kit

Results: The mean age in cases was $31.1 \pm 10.2$ years and mean age in controlwas $34.6 \pm 8.0$ and in control II was $36.3 \pm 10.4$. Urinary MCP-1 values in cases $(1214 \pm 1467.1 \mathrm{pg} / \mathrm{mg})$ was significantly higher compared to Controls $(184.5$ \pm 186.8 . . However, no significant difference was observed between Control (170.5 \pm 150.8$)$ and Control II (194 \pm 214$)$. Urinary MCP-1 levels show significant correlation when compared with classical disease markers like 24 hour proteinuria, 24 hour PCR, Spot PCR and SLEDAl. Cut off value $(339 \mathrm{pg} / \mathrm{mg}, \mathrm{p}<0.001)$ obtained from ROC curve has sensitivity and specificity of $80 \%$ and $92 \%$ respectively. However, there was no significant difference of Urinary MCP-1 levels was observed among different classes of $L N(p=0.593)$. ROC curve comparison of urinary MCP-1 $(A \cup C=0.879)$ with 24 hour urine protein to creatinine ratio ( $A \cup C=0.964)$ and spot urine protein to creatinine ratio $(A \cup C=0.872)$ showed that it is not a better marker of disease activity than both of them. Urinary MCP-1 levels didn't show significant correlation with Renal activity index calculated on renal biopsy.

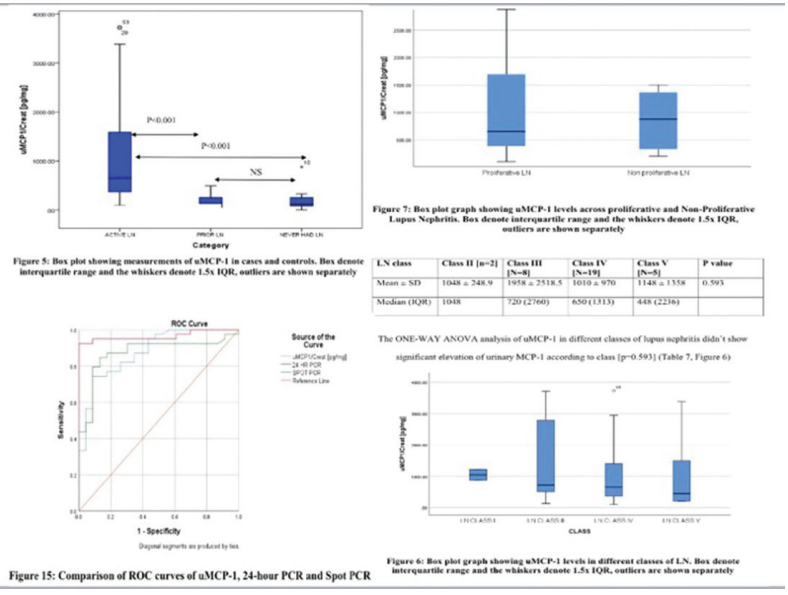

Conclusions: In this study we found higher Urinary MCP-1 levels in active LN compared to Inactive $L N$ and its level was correlated well with the presently used disease markers. However there was no significant difference among different histological classes of LN

Disclosure of Interest: None declared

DOI: 10.1136/annrheumdis-2018-eular.6733

\section{$\mathrm{AB} 1377$}

IS THE PATIENT GLOBAL HEALTH ASSESSMENT RELIABLE IN JUVENILE IDIOPATHIC ARTHRITIS (JIA)?

R. Trachtman, C.M. Wang, J. Szymonifka, K.B. Onel. Hospital for Special Surgery/ Weill Cornell Medicine, New York, USA

Background: JIA is a chronic autoimmune disease that poses many challenges. There is increasing recognition of the importance of patient-reported outcomes (PROs) and newer PROs are being developed and more widely utilised both in clinical care and in research, However, their performance and reliability remain unclear

Objectives: This study seeks to evaluate: ${ }^{1}$ performance of the patient global health assessment (PGA) compared to standard disease activity measures in children with $\mathrm{JIA}{ }^{2}$ correlations of the PGA with socioeconomic status (SES) in JIA and $^{3}$ relationship between $P G A$ and physician global health assessment in JIA. Methods: A convenience sample of patients with JIA $(n=47)$ aged 2-18 were recruited from a single centre. Patients aged $\geq 10$ years completed the questionnaire, and parents of patients aged 2-9 completed a proxy questionnaire for their child. Correlations between ${ }^{1}$ the PGA and disease activity, as measured by the Juvenile Disease Activity Score-71 (JADAS-71), ${ }^{2}$ the PGA and physician global health assessment, and ${ }^{3}$ the physician global health assessment and the JADAS 71 were evaluated using Spearman correlation coefficients. PGAs were compared by age, sex, insurance status, race, and ethnicity; and differences between 
PGA and physician global health assessments were compared using Wilcoxon rank-sum tests.

Results: 16 parents and 31 patients completed the assessments (table 1). There was a moderate correlation between PGA and JADAS-71 ( $r=0.503, p<0.001)$, and $P G A$ and physician global health assessments $(r=0.503, p=0.002)$. There was a stronger correlation between physician global health assessments and JADAS71. PGA median scores and IQRs appeared to be higher among patients with Medicaid insurance, non-white race, and Hispanic ethnicity, with the greatest difference seen in the category of race (Table 2). There were no differences between patient and physician assessments across all groups except among patients with Medicaid (difference median $=-1.25$ ) and Hispanic patients (difference median=2)

Abstract AB1377 - Table 1. Patient Characteristics

\begin{tabular}{lccc}
\hline & $\begin{array}{c}\text { Patients and Parents } \\
(\mathbf{n}=\mathbf{4 7})\end{array}$ & $\begin{array}{c}\text { Patients } \\
(\mathbf{n}=31)\end{array}$ & $\begin{array}{c}\text { Parents } \\
(\mathbf{n}=16)\end{array}$ \\
\hline $\begin{array}{l}\text { Age, years } \\
\text { (median, IQ range) }\end{array}$ & 12.4 & 14.2 & 4.3 \\
Male Gender & {$[8.6-15.0]$} & {$[12.4-15.8]$} & {$[3.3-9.0]$} \\
Race & $17(36.2 \%)$ & $14(45.2 \%)$ & $3(18.8 \%)$ \\
Caucasian/White & & & \\
Asian & $38(80.9 \%)$ & $24(77.4 \%)$ & $14(87.5 \%)$ \\
Other & $3(6.4 \%)$ & $3(9.7 \%)$ & $0(0.0 \%)$ \\
Hispanic Ethnicity & $6(12.7 \%)$ & $4(12.9 \%)$ & $2(12.5 \%)$ \\
Insurance & $8(17.0 \%)$ & $5(16.1 \%)$ & $3(18.8 \%)$ \\
Medicaid & & & \\
Private & $8(17.0 \%)$ & $4(12.9 \%)$ & $4(25.0 \%)$ \\
& $39(83.0 \%)$ & $27(87.1 \%)$ & $12(75.0 \%)$ \\
\hline
\end{tabular}

Abstract AB1377 - Table 2. Comparison of Patient Global Assessments by age, sex, insurance status, race and ethnicity

\begin{tabular}{lcr}
\hline & Patient global median [IQR] & P-value \\
\hline Age & $2[0-5]$ & 0.892 \\
Patient completed & $2[0.05-4.5]$ & \\
Parent completed & $2[0-5]$ & 0.679 \\
Sex & $2[0-4]$ & \\
Male & & \\
Female & $2.5[1,5]$, & 0.313 \\
Insurance & $2[0-4]$ & \\
Medicaid & & 0.266 \\
Private & $1.5[0-4]$ & \\
Race & $4\left[{ }^{2-5}\right]$ & \\
White & $2.5[2.5-5]$ & 0.134 \\
Non-white & $1[0-4]$ & \\
Ethnicity & & \\
Hispanic & Non-Hispanic &
\end{tabular}

Conclusions: Our results demonstrate that physician global health assessment had a stronger correlation with standard disease activity measures than the PGA. These scores were higher in patients who were non-White race, Hispanic, and had Medicaid insurance; however, these were not statistically significant. These data indicate that the PGA is fairly stable across groups, and can be used reliably for disease monitoring.

Disclosure of Interest: None declared

DOI: 10.1136/annrheumdis-2018-eular.7351

\section{AB1378 CLINICAL COMPARISON OF NEW CRITERIA FOR INFLAMMATORY MYOPATHY IN A COLOMBIAN COHORT}

R. Fuentes ${ }^{1}$, D. Gil-Calderon ${ }^{1}$, M. Cañola ${ }^{1}$, J.C. Diaz-Coronado ${ }^{2}$, A. RojasVillarraga $^{2}$, D. Hernandez-Parra ${ }^{2}$, P. Perez-Estrada ${ }^{2}$, J.C. Salazar-Uribe ${ }^{3}$, R. Pineda-Tamayo ${ }^{2} .{ }^{1}$ Rheumatology; ${ }^{2}$ Clinical Research, Artmedica; ${ }^{3}$ National University of Colombia, Medellin, Colombia

Background: Idiopathic inflammatory Myopathies are a group of diseases characterised by weakness due to muscle inflammation, with or without dermatological involvement, that classically includes pathognomonic findings like Gottron sign. There have been different criteria used to classify these diseases, being Peter and Bohan the most used in time, but recently the ARC/EULAR group proposed a new wat to approach the classification.

Objectives: To compare ACR/EULAR 2017 versus Peter and Bohan criteria for Idiopathic Inflammatory Myopathy in a Colombian cohort

Methods: A cross-section retrospective research was done with data collected between 2014 and 2017 from a population diagnosed with Idiopathic Myopathy according to Peter and Bohan criteria and followed up for at least six months. The new ACR/EULAR criteria were applied to each individual using the online tool (hhtp://www.imm.ki.se/biosatistics/calculators/iim). Both sets of criteria were compared using Cohen's kappa coefficient and concordance was evaluated.

Results: Data of 149 patients were obtained. Anti-Jo1 results were not available for $75 \%$ of the patients. Biopsy was available in $44,3 \%$ of patients. Biopsy results were compatible with inflammatory myopathy in $66,7 \%$ and non-compatible in $33,3 \%$. According to Peter and Bohan criteria the diagnosis of idiopathic inflammatory myopathy was definite in $63,1 \%$ of the patients, and probable in $27,5 \%$. Using ACR/EULAR 2017 criteria instead the diagnosis was definite in 63,1\%, probable $10,1 \%$ and non-possible in 20,8\%. According to the new criteria, 31 patients had polymyositis, 47 dematomyositis, 4 amyopathic dermatomyositis, 35 juvenile myositis and 1 inclusion body myositis. The concordance analysis between the two sets of criteria showed agreement of $54 \%$ (kappa $0,22 p<0,001$ ) in the whole group, 59\% (kappa 0,21 $\mathrm{p}<0,001$ ) in adults, 32\% (kappa $0,18 \mathrm{p}=0,05$ ) in children, $44 \%$ (kappa $0,05 \mathrm{p}=0,3$ ) in polymyositis and $42 \%$ (kappa $0,16 \mathrm{p}=0,1$ ) in dermatomyositis.

Conclusions: Data of 149 patients were obtained. Anti-Jo1 results were not available for $75 \%$ of the patients. Biopsy was available in $44,3 \%$ of patients. Biopsy results were compatible with inflammatory myopathy in $66,7 \%$ and noncompatible in $33,3 \%$. According to Peter and Bohan criteria the diagnosis of idiopathic inflammatory myopathy was definite in $63,1 \%$ of the patients, and probable in $27,5 \%$. Using ACR/EULAR 2017 criteria instead the diagnosis was definite in $63,1 \%$, probable $10,1 \%$ and non-possible in $20,8 \%$. According to the new criteria, 31 patients had polymyositis, 47 dematomyositis, 4 amyopathic dermatomyositis 35 juvenile myositis and 1 inclusion body myositis. The concordance analysis between the two sets of criteria showed agreement of 54\% (kappa 0,22 $p<0,001$ ) in the whole group, 59\% (kappa 0,21 p<0,001) in adults, 32\% (kappa 0,18 $\mathrm{p}=0,05$ ) in children, $44 \%$ (kappa $0,05 \mathrm{p}=0,3$ ) in polymyositis and $42 \%$ (kappa 0,16 $\mathrm{p}=0,1)$ in dermatomyositis.

\section{REFERENCE:}

[1] I. Lundberg, et al. 2017 European League Against Rheumatism/American College of Rheumatology classification criteria for adult and juvenile idiopathic inflammatory myopathies and their major subgroups. Ann Rheum Dis 2017:0:1-10. doi:10.1136/annrheumdis-2017-211468

Disclosure of Interest: None declared

DOI: 10.1136/annrheumdis-2018-eular.2189

\section{AB1379 POTENTIAL DIAGNOSTIC SERUM IMMUNOLOGICAL MARKER PANEL IN PRIMARY AND SECONDARY OSTEOARTHRITIS IN SRI LANKAN PATIENTS}

S. Rankotgedera ${ }^{1}$, I. Atukorala ${ }^{2}$, D. Munidasa ${ }^{3}$, L. Wijayaratne ${ }^{4}$, P. Udagama ${ }^{1}$. ${ }^{1}$ Department of Zoology and Environment Sciences, University of Colombo, Sri Lanka, Colombo 3; ${ }^{2}$ Department of Clinical Medicine, University of Colombo, Sri Lanka, Colombo $8 ;{ }^{3}$ Rheumatology and Rehabilitation Hospital, Ragama; ${ }^{4}$ Department of Zoology and Environment Sciences, Nawaloka Hospital PLC, Colombo 2, Sri Lanka

Background: Osteoarthritis $(O A)$ is commonly perceived as a degenerative joint disease but it is now established that inflammation contributes to OA. ThoughOA is categorised into primary and secondary osteoarthritis, most clinicians manage patients of both categories in a similar manner.

Objectives: The current study aimed to identify potential diagnostic markers of primary and secondary OA. Two cohorts of Sri Lankan patients, one with primary $\mathrm{OA}$ and another with secondary $\mathrm{OA}$ were assessed for a selected panel of immunologic mediators i.e. cytokines (TNF- $\alpha, \mathrm{IL}-1 \beta, \mathrm{IL}-6, \mathrm{IL}-10)$ and nitric oxide derivatives (NOx).

Methods: A case control study was conducted with 40 OA patients (Primary, $\mathrm{n}=30$; Secondary, $\mathrm{n}=10$ ), and 60 age and gender matched controls (normal healthy, $n=30$; Systemic Lupus Erythematosus (SLE) disease control, $n=30$ ). The socioeconomic and demographic data were accrued via an interviewer adminis tered questionnaire. Sandwich ELISAs assayed serum cytokine levels, while the 'Griess assay' measured serum NOx levels.

Results: In comparison to healthy controls, OA patients showed significantly higher serum concentrations of all five analytes tested $(p<0.05)$. Patients with primary OA had significantly higher levels of TNF- $\alpha$ and IL-1 $\beta$ and lower level of IL- 6 in serum compared to disease controls (SLE) $(p<0.05)$. secondary OA patients exhibited a significant increase in serum TNF- $\alpha$ and IL-1 $\beta$ and lower serum IL-10 levels compared to disease controls $(p<0.05)$. In the two test groups, the serum levels of TNF- $\alpha$ and IL- 6 were significantly elevated in secondary OA patients $(p<0.05)$. The NOx concentrations between the two test groups was not significantly different. The Th1:Th2 cytokine ratio (TNF- $\alpha: I L-10)$ was significantly higher in secondary OA compared with primary $O A(p<0.05)$.

The Receiver operating characteristic (ROC) curves identified TNF- $\alpha$ and IL-10 as potential diagnostic biomarkers of both primary $\mathrm{OA}$ and secondary $\mathrm{OA}$ compared with healthy controls. IL1- $\beta$ and IL- 6 may be used specifically as diagnostic 
biomarkers for primary and secondary OA, respectively, when compared with healthy individuals. TNF- $\alpha$ is a suitable biomarker for the diagnosis of both primary and secondary OA when compared to SLE patients, whereas IL- 6 and IL-1 $\beta$ were apparent explicit markers of primary $\mathrm{OA}$, while $\mathrm{IL}-10$ was exclusive for secondary OA.

Abstract AB1379 - Table 1. lists the values obtained from the ROC curves for the cytokine panel and NOx for patients clinically diagnosed with primary and secondary osteoarthritis against normal healthy controls.

\begin{tabular}{|c|c|c|c|c|c|c|c|c|}
\hline & \multicolumn{4}{|c|}{ Primary $\mathrm{OA}$} & \multicolumn{4}{|c|}{ Secondary $\mathrm{OA}$} \\
\hline & $\begin{array}{l}\text { AUC } \\
(\%)\end{array}$ & $\begin{array}{l}\text { Cut-off } \\
\text { value }^{*}\end{array}$ & Sensitivity & Specificity & $\begin{array}{c}\text { AUC } \\
(\%)\end{array}$ & $\begin{array}{l}\text { Cut-off } \\
\text { value }^{*}\end{array}$ & Sensitivity & Specificity \\
\hline $\begin{array}{l}\text { TNF- } \\
\alpha\end{array}$ & 88.3 & $>10.8$ & 93.33 & 80.00 & 97.0 & $>28.2$ & 100.00 & 96.67 \\
\hline IL-10 & 88.6 & $>32.67$ & 85.71 & 92.59 & 93.1 & $>32.67$ & 90.00 & 92.59 \\
\hline IL-6 & 59.3 & $>3.98$ & 89.66 & 40.74 & 81.2 & $>12.02$ & 66.67 & 96.15 \\
\hline IL-1 $1 \beta$ & 85.2 & $>1.18$ & 96.43 & 68.42 & 61.4 & $>0.88$ & 77.78 & 57.89 \\
\hline NOx & 72.0 & $>18.89$ & 93.33 & 60.71 & 73.0 & $>18.89$ & 100.00 & 60.71 \\
\hline
\end{tabular}

${ }^{\star}$ For the cytokines, in $\mathrm{pg} / \mathrm{ml}$ and for $\mathrm{NOx}$ in $\mu \mathrm{mol} / \mathrm{l}$

Conclusions: This preliminary study suggests that higher levels of inflammatory cytokines are present in secondary OA compared to primary OA. Furthermore, distinct markers for primary and secondary $O A$ were identified, indivative of the potential for developing different therapeutic agents for the different types of OA. Acknowledgements: The authors acknowledge the University of Colombo, Sri Lanka for funding.

Disclosure of Interest: None declared

DOI: 10.1136/annrheumdis-2018-eular.6602

\section{AB1380 PERIPHERAL NEUROPATHY IN INFLAMMATORY JOINT DISEASES}

Y. Yehudina ${ }^{1}$, O. Syniachenko ${ }^{2}$, T. Bevzenko ${ }^{3} .{ }^{1}$ internal Medicine, Dnepropetrovsk Medical Academy, Dnipro; ${ }^{2}$ Donetsk Medical University, Lyman; ${ }^{3}$ Shupyk National Medical academy, Kyiv, Ukraine

Background: For frequent extra-articular (systemic) manifestations of joints inflammatory diseases are various damage of the nervous system, ${ }^{2}$ and the presence and severity of peripheral neuropathy (PNP) have significance in the clinical course, which incidence among these patients is $5 \%-10 \% .{ }^{1}$ Nevertheless, many clinical and pathogenic aspects of this peripheral neuropathy (PNP) remain obscure.

Objectives: to evaluate the rate and clinical features of PNP in rheumatoid arthritis (RA), chlamydia urogenital reactive arthritis (ReA), psoriatic arthritis $(P A)$ and ankylosing spondylitis (AS), to examine the issues of pathogenetic constructions such changes of the nervous system, define risk factors.

Methods: The study included 416 patients with inflammatory joint diseases, among them 131 RA patients, 101 ReA, 76 PA and 108 AS. The average age of the examined was respectively $45,32,42$ and 38 years, disease duration $-10,4$, 12 and 11 years, male to female ratio $-1: 5,1: 1,1: 2$ and $10: 1$

Results: The frequency of the PNP in RA, ReA, PA and AS was 13\%, 19\%, 24\% and $34 \%$, while its severity in patients with $P A<R e A<R A<A S$, and the same type ratio of motor, sensory and mixed disorders of inflammatory joints diseases, trophic, visceral and vascular vegetative changes, the connexion with the male sex, the activity of arthritis and the presence of tendovaginitis, participation of immune disorders, endothelial dysfunction of blood vessels and changes in physical and chemical rheological viscoelastic properties of blood in the pathogenesis constructions of the nervous system lesions are united around. ReA and PA are different by frequency of hands and feet distal pathology, the AS - by the beginnings of tunnel syndrome. RA tends to impact on the PNP digital arteritis, myositis, eye disease, and Sjogren's syndrome, ReA - on sacroilitit, PA - on exudative form of cutaneous psoriasis, AS - on eye disease, at that, the risk factors for severe course of neuropathy in RA is considered to involve in the process of elbows, $R e A$ - intervertebral and facet joints, PA - wrist, AS - sacroiliac. Guillain-Barré syndrome develops respectively in $3 \%, 4 \%, 5 \%$ and $9 \%$ of patients with RA, PA, ReA and AS, or in $24 \%, 17 \%, 26 \%$ and $27 \%$ cases of PNP, which is closely linked to the presence of tendovaginitis in all inflammatory diseases and severity of articular syndrome, in RA it depends on the presence of hypothyroidism, in ReA - on nephropathy and violations of the heart's electrical conduction, in AS - on osteoporosis, and seropositive for anti-cyclic citrullinated peptide antibody is a risk factor for such peripheral nervous system disorders.

Conclusions: PNP is a relatively common manifestation of inflammatory diseases of the joints, which correlate with clinical and laboratory signs of the disease, and in the future such active detection of the nervous system pathology will be useful for timely follow-up rehabilitation.

\section{REFERENCE:}

[1] Oomatia A., Fang H., Petri M., Birnbaum J. Peripheral neuropathies in systemic lupus erythematosus: clinical features, disease associations, and immunologic characteristics evaluated over a twenty-five-year study period. Arthritis Rheumatol 2014;66(4):1000-1009.

[2] Sampaio-Barros PD. Epidemiology of spondyloarthritis in Brazil. Am. J. Med. Sci 2011;341(4): 287-288.

Disclosure of Interest: None declared

DOI: 10.1136/annrheumdis-2018-eular.3736

\section{Education}

\section{AB1381 FOUR ANNUAL INTERNATIONAL DIFFERENT MEETINGS OF RHEUMATOLOGY: COMPARATION OF THE CONTENTS, ANALYSIS, CHALLENGE AND OPORTUNITIES}

A.J. Pedro ${ }^{1}$, E. Cuevas ${ }^{1}$, C. Abud ${ }^{1}$, R. Moreno ${ }^{1}$, M. Martinez ${ }^{1}$, D. Herrera ${ }^{1}$ E. Acevedo ${ }^{2}$, H. Lopez ${ }^{2}$, D. Ramos ${ }^{2}$, G. Aguilera Barragan², G. Martinez ${ }^{2}$,

T. Luna ${ }^{2}$, E. Santillan ${ }^{2} .{ }^{1}$ Rheumatology; ${ }^{2}$ Hospital Central Ignacio Morones Prieto, San Luis Potosí, Mexico

Background: The medical meetings are a tool to help us be able to escalate and actualize the medical knowledge and their quality is a responsibility of Colleges and Institutions.

Objectives: To assess the academic level of four types of different annual Meeting of Rheumatology

Methods: We used as support information the summaries published in the sup plements of the journal Reumatologia Clinica, SE1 Vol. 12 of February 2016, the supplement SE 1 Vol. 13 of February 2017, the application for electronic media of the ACR/ARHP 2016 of the American Congress of Rheumatology 2016 and the website for abstracts of ,EULAR 2017 dedicated to the surveys presented in the XLIV Mexican Congress of Rheumatology, XLV Mexican Congress of Rheumatology and the 2016 ACR/ARHP Annual Meeting, and EULAR 2017 respectively, of each survey we was obtained information about of the diverse pathologies, the type of trial, content and population referred (adults versus children).

Results: $275,340,3275$ and 4129 were presented in the XLIV Mexican Congress of Rheumatology, XLV Mexican Congress of Rheumatology, the 2016 ACR/ARHP Annual Meeting and EULAR 2017 respectively. Rheumatoid arthritis (RA) was the most common pathology with 23\%, 26\%, 21\% and 27\% in CMR 44, CMR 45, ACR 2016 and EULAR 2017 respectively, followed by systemic lupus erythematosus, third place was vasculitis, beside in international congress was the spondyloartrhitis Highlighted, RA the items about of clinic manifestations were accounted for almost $30 \%$ in the Mexican congress and almost $20 \%$ in ACR and EULAR.

Observational studies accounted for almost $40 \%$ in Mexican congresses vs. $33 \%$ in ACR 2016 and $55 \%$ in, EULAR 2017 beside surveys about of basic research were minimal in Mexican congress, but in ACR 2016 accounted for $21 \%$ and $12 \%$ in EULAR 2016

The trials about of Paediatric Rheumatology were $12.3 \%, 5.5 \%$ and $4.9 \%$ in CMR 44, CMR 45 and ${ }^{\text {ACR } 2016}$ respectively.

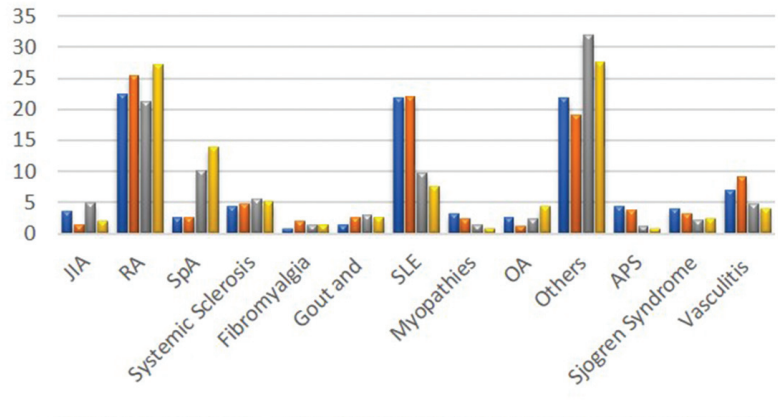

घCMR 44 घCMR 45 घ2016 ACR/ARHP Annual Meeting घEULAR 2017

Abstract AB1381 - Figure 1. percentage of rheumatic disease

Conclusions: Rheumatology Meeting constitutes a support to obtain the adequate medical knowledge based in evidence, in this important branch of 